\title{
Dance as a viable alternative to sport: Effects of traditional dances on the health and fitness of Zimbabwean women
}

\author{
Edmore Nhamo ${ }^{1}$, Simbarashe Magonde ${ }^{2}$ \\ ${ }^{1,2}$ Department of Health Sciences, Faculty of Science and Technology, Zimbabwe Open University, Zimbabwe
}

\begin{abstract}
Dance is corporeal in nature and as such it is a physical art within the domains of anatomy, biomechanics, health, physics and physiology. Dance helps participants develop physical fitness and thus shares many of the health benefits of other sporting events. However in spite of this realization the uptake and adoption of traditional dance is not being prioritized in Zimbabwe. The situation on the ground seems to suggest that the rate of participation in traditional dance has gone down from historical times. This paper discusses the potential and positive effects of traditional dance on the health and fitness of Zimbabwean women. The study uses a mixed method approach and collected data through the use of a battery of tests and interviews. The findings and recommendations provided are expected to encourage Zimbabwean women to appreciate traditional dance as an alternative to organized sport and physical activity. The results indicate that Zimbabwean traditional dances do contribute to the health and fitness of Zimbabwean women. This study recommends that traditional dances should not be trivialized and the Ministry of Sports, Arts and Culture should invest in the promotion of traditional dances in order to complement sport and enhance the health and fitness of Zimbabwean women.
\end{abstract}

Key words: Health and fitness, Sport and Physical activity, Traditional dance, Zimbabwean women

\section{Introduction}

The president of the republic of Zimbabwe recently appointed a new cabinet in September 2013[1]. One of the notable changes in the newly appointed cabinet was the separation of the Ministry of Education, Arts, Sports and Culture into two Ministries viz, the Ministry of Sports, Arts and Culture and the Ministry of Primary and Secondary Education. The major reason for the separation which was welcomed by many stakeholders was to give due prominence to Sports, Arts and Cultural activities which for many decades had played second fiddle to Education in the previous ministry.

Traditional dance is an art and it is also a means of preserving culture. Zimbabwe is rich in traditional/cultural/indigenous dances. From historical times dance has always been a constant source of leisure, inspiration delight and a cultural way of life for Zimbabweans. Zimbabweans dance at weddings, funerals and many other ceremonial and ritual gatherings. As stated by Hanna [2], dance provides an opportunity for expression of aesthetic and artistic experiences and has the capacity to stimulate imagination, sensitivity and the appreciation of movement. Engangement in traditional dance is equvalent to enganging in sport and physical activity, while physical activity and sport is belived to be associated with significant health benefits. Several studies have proved that the human body can be strengthened by physical activity and can also be impaired by physical inactivity [3]. Fox and Mathews [4] , Davis and Butler [5] , and the United Nations [6], all concur that engaging in physical activity is associated with significant health benefits. Physical activity have the potential to reduce the risk of lifestyle or non-communicable diseases such as cardiovascular diseases, cancer, diabetes and conditions such as stress, anxiety and depression[4.5.6]. Physical activity is also believed to help prevent and reduce hypertension, control body weight, prevent and control osteoporosis and help manage chronic pain $[4,5,6]$.

However the positive benefits and value of traditional/ritual/cultural dances as a physical activity and its contribution to health and fitness has not been fully appreciated and acknowledged in Zimbabwe. It is hoped that the findings of this study will encourage the newly created stand alone Ministry of Sports, Arts and Culture to appreciate the significant link between sport and cultural dances and to understand that apart from preserving culture and providing entertainment traditional dances also have positive physiological developmental consequences and implications similar to sport. These can be quite helpful in a nation like Zimbabwe where the healthy delivery system is currently not at its best and where older women generally and characteristically shy away from taking part in sport. The relationship between Zimbabwean traditional dances and the improvement of the health of women is the subject of this investigation. This empirical inquiry is guided by the following research questions: 


\section{Research Questions}

1. Do traditional dances in Zimbabwe meet the requirements of the FITT formula i.e. F = Frequency, I $=$ Intensity, $\mathrm{T}=$ Time/Duration, $\mathrm{T}=$ Type?

2. Do traditional dances contribute to the health related fitness component of the Zimbabwean women i.e. $\mathrm{VO}_{2} \max$ ?

3. To what extend do traditional dances contribute towards other fitness components such as muscular strength, flexibility, muscular endurance and body composition of Zimbabwean women?

\section{Literature Review}

Dance is a human behavior composed of purposeful, intentionally rhythmical, culturally influenced sequences of non-verbal body movements, mostly separate from those performed during ordinary motor activities [2]. The motion in dance has an inherent and aesthetic value. Dance is described as having distinct features which include the intertwined multi-sensory creative imagination, feeling, physical action and language like communication through historical time and across geographical space. Physical movement is the medium of dance and the motor activities may be culturally specific [2]. Dance is corporeal and as such it is a physical art within the domains of anatomy, biomechanics, health, physiology and physics [2]. Dance helps participants develop physical fitness and thus shares many of the health benefits of other athletic activities [2].

\subsection{Cultural dances in Zimbabwe}

Zimbabwean dances have a very distinct, individual style since the tempo of the music and dance is influenced by the spirit of the dancers [7]. Zimbabwean dances can be very powerful and provoke meaning to the performer and the audience [7]. Rhythm is the most important element in Zimbabwean traditional dances. Zimbabwean dances are often characterized by flexed knees, and flat footed, shuffling movements [7]. Other elements include isolation, angularity (with body bend at the waist), asymmetry, improvisation, a swinging quality as well movements outward from the hips [7]. Functions at which dancers perform, last not less than half an hour, and sometimes continue throughout the night as participants take turns to occupy the dance floor. The dances are therefore physiologically demanding [7]. On the whole cultural/indigenous dances involve both moderate to fast movements of the major muscle groups intercepted by similar recovery periods neatly blended together [7]. Traditional dances are characterized by the stamping of feet, swinging of arms, gyrations, warlike gestures and sometimes acrobatics [7].

The traditional/ cultural dances of the Ndebele people can be classified into two major categories, i.e. royal ceremonial and general ceremonial dances [8]. The royal dances include inxwala (first fruits), Ukugcotshwa kweNkosi (royal coronation), umugcabo weNkosi (burial of a king), military rites and the rain making ceremonies [8]. Other ceremonies such as the puberty for boys and girls, marriage, death rites, the bringing home (umbuyiso) and spirit mediums are also characterized by appropriate dances and music and constitute the general ceremonial class of dances [8].

The decline in traditional dances in Zimbabwe started during the colonial era. The indigenous dances were regarded by the white community as primitive, crude, not aesthetic and pure enough. They were generally perceived as barbaric, insignificant and therefore should be jettisoned in favour of the European dance styles. [8] However the traditional dances in their diverse form continued to play a role in the cultural lives of the Zimbabwean people although on a decreased scale [8]. The fusion of cultural dances and modern dances brought about a new dimension which ensured the continuity of Zimbabwean cultural identity [8].

\subsection{Fitness}

According to Siedentop [9 p 169) fitness is viewed as "a series of components each of which is specific in its development and maintenance". There are essentially two categories of fitness components, those particularly related to health and those related to motor - skill performance of specific functional motor tasks [9]. The health related fitness components include, aerobic fitness, muscular strengths, flexibility, muscular endurance and body composition. As emphasised by Siedentop [9] above, fitness is specific and since fitness is specific in its development it is neither expected, nor is it anticipated that traditional dances alone will develop all the fitness components equally. As a result the study will focus on aerobic capacity $\left(\mathrm{VO}_{2} \max \right)$ as the biggest most applicable and most important measure of fitness and health in traditional dances, while all the other components will only play a supportive role.

Aerobic $\left(\mathrm{VO}_{2} \mathrm{max}\right)$ also known as maximal oxygen consumption, maximal oxygen uptake, peak oxygen uptake or maximal aerobic capacity is the maximum capacity of an individual's body to transport and use oxygen during incremental exercise which reflects the physical fitness of the individual [10]. The name $\mathrm{VO}_{2}$ max is derived from "V - Volume", " $\mathrm{O}_{2}$ - Oxygen", "max - maximum" [10]. Willmore and Costill [11] describe aerobic fitness as relating to cardio-vascular endurance, which is the capacity to persist in strenuous activity for long durations which are dependent upon the combined efficiency of the blood vessels, heart and 
lungs. Aerobic exercise/activity is continuous for periods of 20 to 30 minutes and involves the use of large muscle groups, causes stress to the heart, lungs and circulatory system, accelerating their functions and raising the body's metabolic rate [10]. Studies of the relationship between dose and response indicate that cardiovascular health $\left(\mathrm{VO}_{2} \max \right)$ benefits occur at moderate levels of physical activity [12]. Traditional dances are characterised mostly by moderate levels of physical activity.

According to Davis, Butler and Husker [13] Muscular strength is the ability of muscles to exert force, it involves performing an action that requires large amounts of force and can only be completed in a maximum of ten repetitions. Flexibility is the ability of muscles, ligaments, and tendons to stretch so that joints allow movements [13]. Siedentop [9] states that flexibility is the ability of a joint to move through its full range of motion and thus important for health fitness. Flexibility manifests itself in lower risks of injuries during active leisure pursuits. Davis, Butler and Husker [13], refer to muscular endurance as the ability of muscles to produce small efforts that are repeated over and over again.

Body composition is the body's relative amount of fat to fat-free mass [14]. Those with optimal body composition are typically healthier, move more easily and efficiently, and in general, feel better than those with less-than-ideal body composition [14]. Achieving a more optimal body composition goes a long way toward improving your quality of life and overall wellness [14]. According to Siedentop [9], body composition is considered to be an important measure of health fitness. Assessing body mass index (BMI) is a commonly-used method of measuring body fat. While BMI does not measure body fat directly, it helps to assess health risks related to body mass. [14] Ways to assess your body composition, and body fat percentage, more directly include measurement with gun calipers and tests such as underwater body fat test, the Bod Pod, Scan, Biolectrical Impedence Analysis (BIA), Whole-Body Air Displacement (ADP) technology, Dual energy X-ray absorptiometry (DEXA), Magnetic resonance imaging (MRI), Computed tomography(CT) and Ultrasound [14].

\subsection{Health}

The word "health comes from the old English word "Hale" meaning "wholeness, a being whole, sound or well" [15]. Medicalnewstoday.com [15] cite the Medilexion's Medical dictionary as giving three definitions of health, one of which states that " Health is a state of the organism when it functions optimally without evidence of disease or abnomality. This definition does not give a comprehensive description of health. The limitations presented by this as well as by many other definitions led to the development of the most famous, and widely accepted definition of health ie The World Health Orgainisation (WHO) definition. This definition was created during a preamble to the constitution of the World Health Organisation and adopted by the International Health Conference, New York in 1946. The World Health Organisation [16 p1) defines health as a "state of complete physical, mental and social well being and not merely the absence of desease or infirmity". This definition has not been ammended since 1948 and is embraced in this study due to its holistic nature which attempts to define health from a physical, mental and social standpoint. This definition suits sport and physical activity very well which seeks to educate people physically, mentally and socially and thereby contributing to the total health of individuals.

\subsection{Intensity and duration of Excercise}

According to Zakus, Njekesani and Darnell (12 p 17) "It is important to address the question of what constitute an adequate level of intensity and duration to achieve therapeutic benefits from exercise" This as an area of continuous debate but the current public health guidelines suggest that a minimum of 30 minutes per day of moderate physical activity (at approximately $50-60 \%$ of maximum heart rate) is considered optimal [12]. While these guidelines are extremely popular in the biomedical community, they are not as popular in the broader community where they are considered to be unrealistic for many people [12].

Davis, Butler and Husker [17] on the other hand suggest a formula which can be used to dermine the quantity and intensity of exercise for healthy fitness. They call the formula the FITT formula and argue that, to improve fitness one needs to perfom activities that make the heart, lungs and muscles work. They suggest activities like running or swimming and emphasise that the exercise should follow the FITT formula: $F$ for Frequency: they suggest three or four excerscise sessions each week. I for Intensity : to improve heart and lung (aerobic) efficiency, raise pulse to reach 140-160 beats per minute during exercise. $\mathrm{T}$ for time: they suggest exercising continuously for at least 20 minutes inorder to get real benefit. $T$ for type: the suggested best activities are running, cycling, swimming, aerobics, surfing, power walking and many others. The FITT formular, is key to improving the aerobic fitness of an athelete and is the most important formula to remember when persuing an active lifestyle [17]. Reviewed literature reveal that there is widespread debate about what constitude the optimum quantity and intensity, there is no general agreement but there is an ermeging view and generalisation that any level of activity is much better than no activity at all. 


\section{Materials And Methods}

The study was predominantly a quasi- experimental design which utilized a battery of fitness tests to measure fitness components and in depth interviews to collect data on elements of the FITT formula. The design involved the control and active manipulation of variables by way of adhering to correct test protocols and the use of basic testing equipment and facilities. The study purposively sampled three traditional dance groups in Bulawayo metropolitan province. The total number of dance groups in Bulawayo was not ascertained. Even though the dance groups were made up of both man and women the study only focused on the 50 women who were members of the three dance groups which were purposively sampled.

\subsection{Measurement protocols}

\subsubsection{Intensity of training: Target heart rate}

Measuring pulse rate after exercise gives a good indication of how efficiently one's heart and lungs are working. The rate at which one's heart should be working during continuous training session is known as your target heart rate [5]. Because this varies with age, a target heart rate was worked out for each of the subjects in this study. First the maximum heart rate was determined; this was done by subtracting each Subject's age from 220. The target heart rate was then worked out by calculating 75 percent of participants' maximum heart rate. For example, The determination of the target heart rate of a 16 year old girl was done as follows:

Maximum heart rate $=220$ - age (beats per minute)

Maximum heart rate $=220-16=204 \mathrm{bpm}$ (beats per minute)

Target heart rate $=$ maximum heart rate $\times 75 \%$ (beat per minute)

Target heart rate $=204 \times 75 \%=153$

\section{Target zone}

Target zone is the range in which the body should be working. This zone generally ranges from 70 per cent to 85 per cent of maximum heart rate. Therefore a 16 year - old should have a heart rate roughly between 143 and 173. To check this the subjects were asked to pause during a session and either their radial pulse or your carotid pulse for 30 seconds was taken. This count was doubled to give each subject's one-minute heart rate.

Evaluation

If heart rate was below 143 the subject was considered not to be working hard enough

If heart rate was above 173 the subject was considered to be working too hard

Source of measurement protocol: Davis and Butler [5]

\subsubsection{Aerobic capacity: $\mathrm{VO}_{2} \max$}

The Harvard step test was used as a measurement protocol to measure aerobic capacity and was considered the most important test in this study. It was administered as follows:

Equipment

* For girls below 17 and women between 35 - 55 a $41 \mathrm{~cm}$ high bench was used

* For women between $18-35$ a $46 \mathrm{~cm}$ high bench was used

* For women above 55 years a $35 \mathrm{~cm}$ high bench was used

* Stop watches with second hands were used and

* Score sheets

Method

1. The subjects exercised on the appropriate benches at the rate of 30 steps per minute for five minutes for adults and three minutes for children and elderly people.

2. A full cycle represented a 4-count beat

$\checkmark$ On count 1 , the subject places one foot on the step

$\checkmark$ On count 2, the other leg is lifted onto the step and the subject straightens both legs

$\checkmark$ On count 3, the lead foot is brought down

$\checkmark$ On count 4 , the second foot follows

Recording

1. At the end of the test, the subject immediately sat down, and the duration of the exercise was recorded.

2. The pulse of the subject was counted from:
$\checkmark \quad 1-1.5$ minutes post exercise
$\checkmark \quad 2-2.5$ minutes post exercise
$\checkmark \quad 3-3.5$ minutes post exercise

The sum of pulses during the three 30 -s period was recorded 
Calculations

The scores were determined by the following equation:

$\checkmark \quad$ Index $=\underline{100 \times \text { (duration of exercise in seconds) }}$

$2 \times$ (sum of pulses)

The scores were then classified using the Harvard step test fitness index below:

Table 1: Harvard step test fitness index

\begin{tabular}{|l|l|}
\hline Index & Classification \\
\hline$>90$ & Excellent \\
\hline $80-89$ & Good \\
\hline $65-79$ & High average \\
\hline $55-64$ & Low average \\
\hline$<55$ & Poor \\
\hline
\end{tabular}

Validity: Correlation to $\mathrm{VO}_{2}$ max approximately 0.6 to 0.8

Source: Kanhukamwe and Tapera [18]

\subsection{The FITT formula}

\section{Results}

\subsubsection{F: Frequency of Training}

Number of practice sessions

Data collected on the number of practice sessions (i.e. frequency of training) of the participants from the study indicated that 40 of participants $(80 \%)$ practice three or more times every week. Ten of the participants $(20 \%)$ attended practice sessions less than three times a week.

Number of performances to audience

Since performance is based on invitations it is varied, however according to results obtained on the number/frequency of performances to audience the subjects indicated that twenty five $(50 \%)$ percent perform more than once a week. The other twenty five (50\%) perform on average once every two weeks, sometimes more and sometimes less frequently.

\subsubsection{I: Intensity of training}

Table: 2 Participants' Target heart rate

\begin{tabular}{||l||l||l|l||}
\hline \hline Target heart rate & Frequency & \% & Classification \\
\hline \hline Below 143 & 10 & 20 & Too low \\
\hline \hline Between $143-173$ & 35 & 70 & Ideal \\
\hline \hline Above 173 & 5 & 10 & Too hard \\
\hline \hline Total & $\mathbf{5 0}$ & $\mathbf{1 0 0}$ & \\
\hline \hline
\end{tabular}

$\mathrm{n}=50$

The target heart rates of participants were measured during practice. It was found that seventy percent $(70 \%)$ had a target rate between 143 and $173 \mathrm{bpm}$. Ten $(20 \%)$ had a target heart rate below $143 \mathrm{bpm}$. Five of the participants $(10 \%)$ recorded a target heart rate which was more than $173 \mathrm{bpm}$.

The target heart rate was also measured during selected performances and thirty eight (76\%) of the participants recorded a target heart rate between 143 and 173 . Twelve $(24 \%)$ recorded a target heart rate above 173 while none $(0 \%)$ were below 143 .

\subsubsection{T: Time/duration of training}

Dancers indicated that they perform for at least one hour and practice for at least thirty minutes or more. However longer sessions were intercepted by breaks as dancers take turns to perform and also take frequent coaching and resting breaks during training sessions.

\subsubsection{T: Type/mode of activity}

The older women who constituted $40 \%$ concentrated on cultural dances of the Ndebele origin only. The younger groups of women i.e. $60 \%$ were more versatile and performed almost all Zimbabwe cultural dances as well as dances from other regions. Observations and measurement of target heart rate indicated that the type of activity was predominantly aerobic in nature and bordered around interval training as the dances were never continuous but intercepted by regular breaks.

\subsection{Fitness components test results}

5.2.1 Aerobic capacity $\mathrm{VO}_{2} \max$ (Estimation of aerobic capacity) 
Table 3 the Harvard step test

\begin{tabular}{|l||l||l||l||}
\hline \hline Index & Frequency & $\mathbf{\%}$ & Classification \\
\hline \hline$>90$ & 18 & 36 & Excellent \\
\hline \hline $80-89$ & 11 & 22 & Good \\
\hline \hline $65-79$ & 15 & 30 & High average \\
\hline \hline $55-64$ & 3 & 6 & Low average \\
\hline \hline$<55$ & 3 & 6 & Poor \\
\hline \hline Total & 50 & 100 & \\
$\mathrm{n}=50$ & &
\end{tabular}

Eighteen of the women (36\%) who underwent the Harvard step test were in the excellent category. Eleven $(22 \%)$ were classified as good. Fifteen of the women $(30 \%)$ were on the high average. Three of the women $(6 \%)$ recorded a low average $\mathrm{VO}_{2} \max$, while another three $(6 \%)$ recorded poor. In total forty four of the woman $(88 \%)$ were above the high average score, while a total of twelve $(24 \%)$ were below the high average (low average and poor).

\subsubsection{Strength and muscular endurance}

Table 4 abdominal muscle strength and endurance

\begin{tabular}{|c|c|c|c|c|c|c|c|c|c|}
\hline \multirow[t]{3}{*}{ Fitness test } & \multirow[t]{3}{*}{ Fitness component } & \multicolumn{8}{|c|}{ Ratings } \\
\hline & & \multicolumn{2}{|c|}{$\mathrm{R}$} & \multicolumn{2}{|c|}{$\mathrm{P}$} & \multicolumn{2}{|c|}{$\overline{\mathrm{OOK}}$} & \multicolumn{2}{|r|}{$\mathrm{E}$} \\
\hline & & No & $\%$ & $\mathrm{~N}$ & $\%$ & No & $\%$ & No & $\%$ \\
\hline Sit up & Strength \& muscular endurance & 20 & 40 & 15 & 30 & 10 & 20 & 5 & 10 \\
\hline
\end{tabular}

$\mathrm{n}=50$

Key to fitness ratings (applies to all tables and figures below)

1. $\mathrm{E}=$ Excellent

2. $\mathrm{Ok}=$ Not a bad result

3. $\mathrm{P}=$ Problem area

4. $\mathrm{R}=$ Risky

In order to measure the abdominal muscle strength and endurance of the women, sit ups were used. The Table shows that female dancers were generally in the lower extreme of abdominal muscle strength and endurance. The table of results shows that twenty of the traditional dancers $(40 \%)$ percent were in the risky category. Fifteen $(30 \%)$ were rated as poor while ten $(20 \%)$ and five dancers $(10 \%)$ scored ok and Excellent respectively. A total of thirty five (70\%) were on the weak side while a total of fifteen were $(30 \%)$ were on the strong side of abdominal muscle strength and endurance.

Table 5: Upper body muscles (arms and chest) Strength and endurance

\begin{tabular}{|c|c|c|c|c|c|c|c|c|c|}
\hline \multirow[t]{3}{*}{ Fitness test } & \multirow{3}{*}{$\begin{array}{l}\text { Fitness component } \\
\end{array}$} & \multicolumn{8}{|c|}{ Rating } \\
\hline & & \multicolumn{2}{|l|}{$\begin{array}{l}\mathrm{R} \\
\end{array}$} & \multicolumn{2}{|l|}{$\bar{P}$} & \multicolumn{2}{|c|}{ OK } & \multicolumn{2}{|l|}{$\overline{\mathrm{E}}$} \\
\hline & & No & $\%$ & No & $\%$ & No & $\%$ & No & $\%$ \\
\hline Push up & $\begin{array}{lll}\begin{array}{l}\text { Strength } \\
\text { endurance }\end{array} & \& & \text { Muscular }\end{array}$ & 10 & 20 & 18 & 36 & 17 & 34 & $\overline{55}$ & 10 \\
\hline
\end{tabular}
$\mathrm{n}=50$

To collect data on the upper body muscle strength and endurance of the subjects modified press ups were used. The traditional dancers were required to support their bodies using knees instead of the usual toes (as used in standard press ups). The data in the table indicates that ten $(20 \%)$ of the Traditional dancers' scores were in the risky category on press ups. Eighteen (36\%) were poor, seventeen (34\%) were OK while 5 traditional dancers $(10 \%)$ showed excellent upper body strength and endurance. In total twenty eight $(56 \%)$ did poorly while a total of twenty two constituting (44\%) performed well.

\subsubsection{Flexibility}

Table 6: Flexibility of lower back and the hamstrings

\begin{tabular}{|c|c|c|c|c|c|c|c|c|c|}
\hline \multirow[t]{3}{*}{ Fitness test } & \multirow[t]{3}{*}{ Fitness component } & \multicolumn{7}{|c|}{ Ratings } & \\
\hline & & \multicolumn{2}{|l|}{$\overline{\mathrm{R}}$} & \multicolumn{2}{|l|}{$P$} & \multicolumn{2}{|l|}{$\overline{\mathrm{OK}}$} & \multicolumn{2}{|l|}{$\mathrm{E}$} \\
\hline & & No & \%\% & No & $\%$ & No & $\%$ & No & \%\% \\
\hline Sit \& reach & Flexibility & 3 & 6 & 4 & 8 & 25 & 50 & 18 & 36 \\
\hline
\end{tabular}


Data shows that only three of the fifty women (6\%) were in the Risky category of flexibility. Four $(8 \%)$ were in the problem area. Twenty five $(50 \%)$ were OK while eighteen $(36 \%)$ had excellent flexibility. A total of seven $(14 \%)$ showed low flexibility, while a total of forty three $(86 \%)$ showed high flexibility.

\subsubsection{Strength and Power}

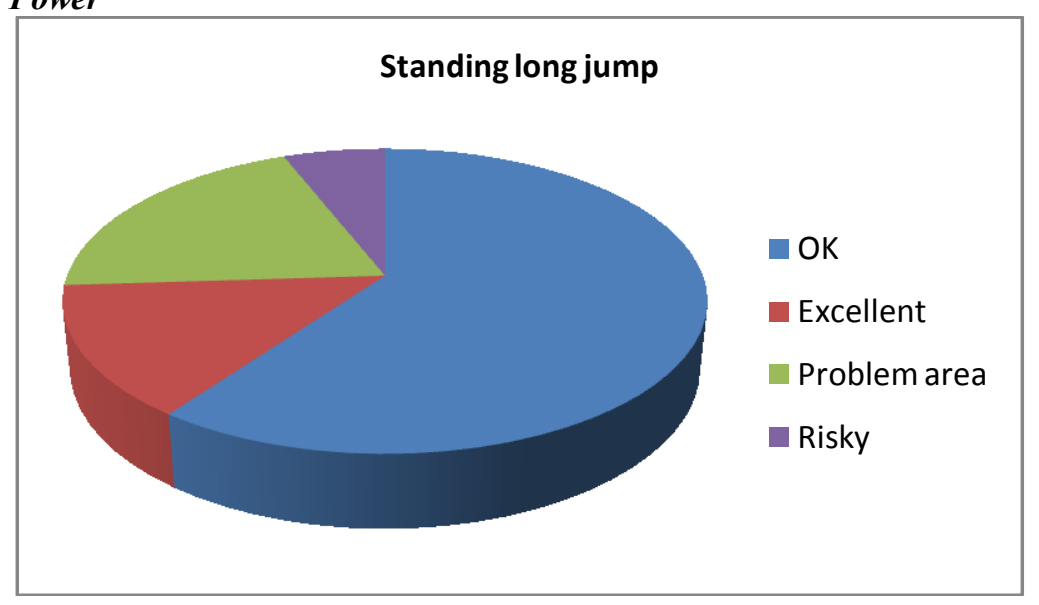

Figure 1: Lower body strength and power

In terms of lower body strength and power the majority i.e. thirty of the Traditional dancers $(60 \%)$ were in the OK category. Seven of the traditional dancers (14\%) were in the excellent category, while ten $(20 \%)$ were in the problem area. Only three $(6 \%)$ were in the risky category. In total thirty seven $(72 \%)$ showed average to above average lower body strength, while thirteen $(26 \%)$ showed below average lower body strength.

\subsubsection{Body composition}

Table 7 Body Mass Index

\begin{tabular}{|c|c|c|c|c|c|c|c|c|c|}
\hline \multirow[t]{3}{*}{ Fitness test } & \multirow[t]{3}{*}{ Fitness component } & \multicolumn{7}{|c|}{ Ratings } & \\
\hline & & \multicolumn{2}{|c|}{$\bar{R}$} & \multicolumn{2}{|c|}{$\overline{P \mathrm{P}}$} & \multicolumn{2}{|c|}{ "OK } & \multicolumn{2}{|c|}{$\bar{E}$} \\
\hline & & No & \%\% & No & $\%$ & No & $\%$ & "No & $\%$ \\
\hline BMI & Body composition & 15 & 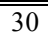 & 21 & 42 & 10 & 20 & 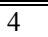 & 8 \\
\hline
\end{tabular}

The Body Mass Index (BMI) was used to measure the body composition of the women traditional dancers. The BMI measurements revealed that fifteen Traditional Dancers (30\%) had a risky BMI. Twenty one $(42 \%)$ had problematic body composition (BMI). However ten of the Traditional dancers $(20 \%)$ were in the ok category while four $(8 \%)$ had an excellent BMI. In total seventy two percent $(72 \%)$ had an unsafe body composition (BMI), while twenty eight percent $(28 \%)$ were on the safe side.

\subsection{FITT Formula}

\section{Discussion}

The main purpose of this study was to investigate the contribution of traditional dances on the health and fitness of Zimbabwean women. The results clearly indicated that the traditional dances met the requirements of the FITT formula in terms of frequency, intensity, type and duration. The dance groups practiced at least 3 times per week which is the average recommended frequency for any physical activity according to the FITT formula. This is believed to bring about improvements on the health and fitness of participants. Davis, Butler and Hasker [17] support this frequency and recommend taking part in three or four exercise sessions each week. If performances are combined with practice session they constitute adequate or even higher frequencies which conform to the requirements of the FITT formula.

In terms of training/practice intensity the traditional dances also met the intensity threshold required by the FITT formula as suggested by Davis, Butler and Hasker [17] who proposed that to improve your heart and lung (aerobic) efficiency, your pulse rate must reach 140 - 160 beats per minute during your exercise and the target heart rate should be between 70 - 85 percent of maximum heart rate. Alternatively Zakus, Njekesani and Darnell [12 p17) suggest a slightly lower target heart rate when they say, that "approximately 50 - 60 percent of maximum heart rate is considered optimal".

The average length of both practice sessions and performances observed constituted adequate duration to meet the FITT formula. Zakus, Njekesani and Darnell [12 p17 refer to the current public health guidelines which suggest that "a minimum of 30 minutes per day of moderate physical activity (at approximately 50-60\% 
of maximum heart rate) is considered optimal". Davis Butler and Hasker [17], posits that to get any real benefit , you must exercise for at least 20 minutes and continuosly with no rests. However research has shown that this remains an area of continuous debate with an emerging view that any activity is better than no activity at all.

Both observation and pulse rate measurements showed that the activities involved in the dances were predominantly aerobic in nature i.e. the dancers were consistently operating at $70-85$ percent of maximum heart rate. This is believed to contribute to the aerobic capacity of the dancers in line with earlier research and literature. Specifically, studies of the relationship between dose and response indicate that cardiovascular health benefits occur at moderate levels of physical activity [12].

\subsection{Fitness Components}

In terms of fitness components the majority of the traditional dancers did well and scored highly on aerobic capacity $\left(\mathrm{VO}_{2} \mathrm{max}\right)$. These positive results can be attributed to the nature of the traditional dances which were mostly of moderate to high intensity and of fairly long durations. As observed by Nyathi [7], Ritual dances last even twelve hours thus are physiologically demanding. This duration of the dances was enough to stimulate the improvement of their aerobic capacity $\left(\mathrm{VO}_{2} \mathrm{max}\right)$. This observation was made in both practice and performance sessions. What helped is that it is mainly the large muscle groups (legs and arms) that are in action for prolonged periods and these demand uptakes of oxygen. The actual dances also take hours of performance intercepted by periods of rest depicting interval training. As Nyathi [7], pointed out, on the whole cultural/indigenous dances involve both moderate to fast movements of the major muscle groups intercepted by similar recovery periods neatly blended together. Dancers rehearse at least three times a week and perform at shows on average once every week. These dances therefore meet the conditions that are recommended for improving and maintaining health fitness. The results on $\mathrm{VO}_{2} \max$ are also duly supported by results on the intensity of dances discussed above. It was observed that the older dancers had poor abdominal muscle strength and endurance as compared to their younger counterparts. This is probably because as women grow older their abdominal muscles get weaker and weaker probably exacerbated by child birth and lack of specific abdominal muscle exercise. The relatively lower than average performance on the upper body muscle strength and muscular endurance could be attributed to smaller shoulders for women and also due to the fact that pushups are generally difficult for women to execute. Also traditional dances do not contribute much towards upper body strength since dancers use their legs mostly to carry their body weight. Traditional dances usually involve swinging of arms as reflected by Nyathi [7], in his description of dance i.e. "traditional dances are characterized by the stamping of feet, swinging of arms, gyrations, warlike gestures as well as acrobatics".

The majority of the subjects performed well on Flexibility. This is because women are physiologically and naturally flexible by nature as compared to their male counterparts and that the traditional dances also helped to enhance their flexibility.

The female dancers performed well in the standing long jump as shown by the results. The good performance could be attributed to the fact that most dances involve stamping of feet and acrobatics which tend to strengthen their lower body muscles. Observations also indicated that some of the dances involved constant vigorous jumping up and down movements. This could have helped develop the lower body strengths of the dancers and hence assist them to overcome their endomorphism.

The results of the body composition i.e. Body Mass Index need to be interpreted with caution because of the widely reported inherent weaknesses of this measurement protocol. Zakus et al [12], and Fox and Mathews [4] noted that there is a lack of clarity in defining obesity and there is also an associated problem of measurement, with concerns being raised about the lack of accuracy of the popular Body Mass Index (BMI). However BMI was used in this study because according to [14] while BMI does not measure body fat directly, it helps to assess health risks related to body mass. The poor ratings obtained in this study could also be related to the body structures/ somatotyping of women. Women put on a lot of weight as they grow older especially after bearing children therefore the proportion between weight and height could be misleading. Despite their heavy weightiness their body shapes were regular and the muscles were particularly distinct in the lower limps. It therefore maybe argued that their apparent endomorphism does not necessarily mean that they are obese, too fat and therefore unhealthy.

\section{Conclusion And Recommendations}

The test protocols administered and the Interviews conducted in this study provided strong evidence that the traditional dances adequately meet the FITT formula. Similarly the data gathered revealed that the dances contribute to the aerobic capacity $\mathrm{VO}_{2} \max$ of the Zimbabwean women. It can therefore be concluded that judging from the fit formula and measurements of critical fitness components, traditional dances contribute to the health and fitness of Zimbabwean women especially the cardiovascular fitness/ aerobic fitness $\left(\mathrm{VO}_{2} \mathrm{max}\right)$ which was the most important health component in this study.

It is therefore recommended that the recently created stand alone Ministry of Sports, Arts and Culture should not neglect but go all out to promote participation of women in traditional/indigenous dances in order to 
complement sport in improving the health and fitness of Zimbabwean women. This is especially applicable to those Zimbabwean women who consider themselves too old to take part in recreational or competitive sport due to cultural beliefs.

\section{References}

[1] The Herald, 10 September 2013 (cited 2013 September 10) available from http://www.herald.co.zw

[2] Hanna J, L. Partnering Dance and Education: Intelligent moves for changing times. USA: Human Kinetics; 1999

[3] Almond L. The place of Physical Education, London: Kogan page;1 989

[4] Fox E,L and Mathews D,K. Physiological basis of physical Education and Athletics ( $3^{\text {rd }}$ Edition), Philadelphia: Saunders College of publishing; 1981

[5] Davis D and Butler, T. Health \& Physical Education (Book 2). Australia, Macmillan Education; 1997

[6] United Nations. Sport for Development and Peace: Towards Achieving the Millenium Development Goals , report from the the United Nations Inter-Agency Task Force on Sport for Development and Peace; 2003

[7] Nyathi, P. Sunday news magazine, Bulawayo; 2010

[8] Nyathi, P. Traditional Ceremonies of Amandebele, Mambo press: Harare. 2001

[9] Siedentop, D. Introduction to Physical Education Fitness and Sport ( $5^{\text {th }}$ Edition) New York: Mc Graw Hill High Education; 2004

[10] http://en.wikipedia.org/wiki/vo2max

[11] Willmore, J, H and Costill, D, L. Physiology of Sport and Exercise ( $3^{\text {rd }}$ edition) USA: Human Kinetics; 2004

[12] Zakus, D., Njekesani, D. and Darnell, S. (2007). The use of sport and Physical Acivity to achieve Health Ojectives: Literature Reviews on Sport for Development and Peace International Working Group (SDPIWG), University of Toronto: Faculty of physical Education and Health (cited 2011 Februaly 26) Available from http://www.Litrature reviews.SDP

[13] Davis D, Butler and Hasker L (2000) Health and Physical Education (Book 1) McMillan Education: Australia

[14] http://weightloss.about.com/od/backtobasics/f/bodycomp.htm

[15] Medicalnewstoday. Health. (cited 2011 December 2) Available from http://www.medicalnewstoday.com

[16] World Health Organisation. Exercise for Health: WHO/FIMS Committee on Physical Activity for Health. Bulletin of the World Health Organisation; 1995

[17] Kanhukamwe O and Tapera E. Testing Measurement and Evaluation in PES. Mt Pleasant: Zimbabwe Open University; 2004 